\title{
REALISASI UNTUK SISTEM DESKRIPTOR LINIER INVARIANT WAKTU
}

\author{
NOVRIANTI \\ Program Studi Magister Matematika, \\ Fakultas Matematika dan Ilmu Pengetahuan Alam, Universitas Andalas, \\ Kampus UNAND Limau Manis Padang, Indonesia, \\ rian_ccb@yahoo.co.id
}

\begin{abstract}
A continuous invariant time linear control system is a model which is often applied in economics, engineering, and numerical analysis. In this paper, the realization of the transfer function for the descriptor system with multi inputs and multi outputs (MIMO) is discussed. Some algebraic techniques are used to prove some theorems and to get a realization of a transfer function.

Kata Kunci: Realizations, descriptor system, MIMO, strictly proper
\end{abstract}

\section{Pendahuluan}

Jika diberikan suatu sistem kontrol, maka bukan merupakan hal yang sulit untuk mendapatkan fungsi transfernya, tetapi tidak sebaliknya. Karakteristik hubungan antara input dan output dari suatu sistem kontrol biasanya dapat dilihat dari fungsi transfer. Fungsi transfer untuk suatu sistem kontrol linier invariant waktu didefinisikan sebagai perbandingan antara transformasi Laplace output dengan transformasi Laplace input dengan mengasumsikan syarat awal adalah nol [1]. Penentuan realisasi dari suatu fungsi transfer tidaklah mudah, terutama dalam sistem deskriptor dengan multi input dan multi output (MIMO). Oleh karena itu, kajian tentang realisasi masih sangat diperlukan.

\section{Sistem Deskriptor Linier Invariant Waktu}

Sistem deskriptor linier invariant waktu ditulis sebagai berikut

$$
\begin{aligned}
E \dot{\mathbf{x}}(t) & =A \mathbf{x}(t)+B \mathbf{u}(t), \quad \mathbf{x}(0)=\mathbf{x}_{0} \\
\mathbf{y}(t) & =C \mathbf{x}(t)
\end{aligned}
$$

dimana $\dot{\mathbf{x}}(t)=\frac{d \mathbf{x}(t)}{d t}$. Dalam sistem $(2.1), \mathbf{x}(t) \in \mathbb{R}^{n}$ menyatakan vektor keadaaan, $\mathbf{u}(t) \in \mathbb{R}^{m}$ menyatakan vektor input (kontrol), $\mathbf{y}(t) \in \mathbb{R}^{p}$ menyatakan vektor output, $A, E \in \mathbb{R}^{n \times n}, B \in \mathbb{R}^{n \times m}$, dan $C \in \mathbb{R}^{p \times n}$. Fungsi transfer dari sistem (2.1) adalah $T(s)=C(s E-A)^{-1} B$. Jika matriks $E$ adalah non singular, maka sistem (2.1) merupakan sistem kontrol standar, akan tetapi jika matriks $E$ singular, maka sistem (2.1) harus didekomposisikan dengan menggunakan teorema berikut. 
Teorema 2.1. [1] Sistem deskriptor (2.1) adalah regular jika dan hanya jika terdapat matriks nonsingular $Q, P \in \mathbb{R}^{n \times n}$ sedemikian sehingga

$$
Q E P=\left[\begin{array}{cc}
I_{n_{1}} & 0 \\
0 & N
\end{array}\right] \text { dan } Q A P=\left[\begin{array}{cc}
A_{1} & 0 \\
0 & I_{n_{2}}
\end{array}\right]
$$

dimana $A_{1} \in \mathbb{R}^{n_{1} \times n_{1}}, N \in \mathbb{R}^{n_{2} \times n_{2}}, n_{1}+n_{2}=n$, dan matriks $N$ adalah nilpoten dengan indeks nilpotensi $q$.

Berdasarkan Teorema 2.1, dapat dilihat bahwa matriks $E, A \in \mathbb{R}^{n \times n}$ didekomposisikan sebagai

$$
E=Q^{-1}\left[\begin{array}{cc}
I_{n_{1}} & 0 \\
0 & N
\end{array}\right] P^{-1}, \operatorname{dan} A=Q^{-1}\left[\begin{array}{cc}
A_{1} & 0 \\
0 & I_{n_{2}}
\end{array}\right] P^{-1}
$$

untuk suatu matriks nonsingular $P, Q \in \mathbb{R}^{n \times n}$.

Perhatikan kembali sistem (2.1). Selanjutnya misalkan $\mathbf{x}(t)=P \mathbf{z}(t), Q B=$ $\left[\begin{array}{c}B_{1} \\ B_{2}\end{array}\right]$, dan $C P=\left[C_{1} C_{2}\right]$ untuk suatu $Q, P$ nonsingular, $\mathbf{z}(t) \in \mathbb{R}^{n}, B_{1} \in$ $\mathbb{R}^{n_{1} \times m}, B_{2} \in \mathbb{R}^{n_{2} \times m}, C_{1} \in \mathbb{R}^{p \times n_{1}}$, dan $C_{2} \in \mathbb{R}^{p \times n_{2}}$. Maka persamaan keadaan pada sistem (2.1) dapat ditulis menjadi

$$
\begin{aligned}
E P \dot{\mathbf{z}}(t) & =A P \mathbf{z}(t)+B \mathbf{u}(t) \\
Q E P \dot{\mathbf{z}}(t) & =Q A P \mathbf{z}(t)+Q B \mathbf{u}(t) \\
& =Q A P \mathbf{z}(t)+\left[\begin{array}{l}
B_{1} \\
B_{2}
\end{array}\right] \mathbf{u}(t),
\end{aligned}
$$

dan persamaan output ditulis menjadi

$$
\mathbf{y}(t)=\left[C_{1} C_{2}\right] \mathbf{z}(t) .
$$

Dengan menggunakan Teorema 2.1, maka sistem (2.1) dapat ditulis menjadi

$$
\begin{aligned}
{\left[\begin{array}{cc}
I_{n_{1}} & 0 \\
0 & N
\end{array}\right]\left[\begin{array}{l}
\dot{\mathbf{z}}_{1}(t) \\
\dot{\mathbf{z}}_{2}(t)
\end{array}\right] } & =\left[\begin{array}{cc}
A_{1} & 0 \\
0 & I_{n_{2}}
\end{array}\right]\left[\begin{array}{l}
\mathbf{z}_{1}(t) \\
\mathbf{z}_{2}(t)
\end{array}\right]+\left[\begin{array}{l}
B_{1} \\
B_{2}
\end{array}\right] \mathbf{u}(t) \\
\mathbf{y}(t) & =\left[\begin{array}{ll}
C_{1} & C_{2}
\end{array}\right]\left[\begin{array}{l}
\mathbf{z}_{1}(t) \\
\mathbf{z}_{2}(t)
\end{array}\right] .
\end{aligned}
$$

Persamaan (2.3) memperlihatkan bahwa persamaan keadaan pada sistem (2.1) dapat direduksi menjadi dua subsistem, yaitu

$$
\begin{aligned}
\dot{\mathbf{z}}_{1}(t) & =A_{1} \mathbf{z}_{1}(t)+B_{1} \mathbf{u}(t) \\
N \dot{\mathbf{z}}_{2}(t) & =\mathbf{z}_{2}(t)+B_{2} \mathbf{u}(t),
\end{aligned}
$$

dan persamaan (2.4) memperlihatkan bahwa persamaan output pada sistem (2.1) dapat direduksi menjadi dua subsistem pula, yaitu

$$
\begin{aligned}
& \mathbf{y}_{1}(t)=C_{1} \mathbf{z}_{1}(t) \\
& \mathbf{y}_{2}(t)=C_{2} \mathbf{z}_{2}(t),
\end{aligned}
$$

dimana $\mathbf{z}_{1}(t) \in \mathbb{R}^{n_{1}}, \mathbf{z}_{2}(t) \in \mathbb{R}^{n_{2}}, C_{1} \in \mathbb{R}^{p \times n_{1}}$ dan $C_{2} \in \mathbb{R}^{p \times n_{2}}$, dengan $\mathbf{y}(t)=$ $\mathbf{y}_{1}(t)+\mathbf{y}_{2}(t)$. 
Untuk sistem dengan multi input dan multi output (MIMO), fungsi tansfer didefinisikan sebagai fungsi $T(s)$ yang memenuhi hubungan

$$
\mathbf{Y}(s)=T(s) \mathbf{U}(s)
$$

dengan syarat awal $\mathbf{x}(0)=\mathbf{0}$, dimana $\mathbf{Y}(s)$ adalah transformasi Laplace output dan $\mathbf{U}(s)$ adalah transformasi Laplace input.

Definisi 2.1. [3] Suatu fungsi transfer $T(s)$ dikatakan proper jika $\lim _{s \rightarrow \infty} T(s)=$ $K, K \in \mathbb{R}^{p \times m}$, dan dikatakan strictly proper jika $\lim _{s \rightarrow \infty} T(s)=0$.

\section{Realisasi untuk Sistem Deskriptor Linier Invariant Waktu}

Jika diberikan suatu fungsi transfer $T(s)$, maka fungsi transfer dapat didekomposisikan sebagai

$$
T(s)=G(s)+F(s)=C_{1}\left(s I-A_{1}\right)^{-1} B_{1}+C_{2}(s N-I)^{-1} B_{2} .
$$

dimana $G(s)$ merupakan bagian strictly proper, dan $F(s)$ bagian polinomial.

Teorema 3.1. [2] Realisasi dari $G(s)$ ada jika dan hanya jika $G(s)$ merupakan fungsi transfer yang strictly proper.

$\operatorname{Bukti}(\Rightarrow)$ Misalkan realisasi dari $G(s)$ ada, sebutlah realisasi tersebut adalah $A_{1}, B_{1}, C_{1}$ sedemikian sehingga $G(s)=C_{1}\left(s I-A_{1}\right)^{-1} B_{1}$. Akan ditunjukkan bahwa $G(s)$ merupakan fungsi transfer yang strictly proper. Perhatikan bahwa

$$
G(s)=C_{1}\left(s I-A_{1}\right)^{-1} B_{1}=\frac{C_{1}\left(\operatorname{adj}\left[s I-A_{1}\right]\right) B_{1}}{\operatorname{det}\left(s I-A_{1}\right)} .
$$

Karena derajat entri-entri dari $a d j\left[s I-A_{1}\right]$ selalu lebih kecil dari derajat $\operatorname{det}(s I-$ $\left.A_{1}\right)$, maka

$$
\lim _{s \rightarrow \infty} G(s)=0
$$

Jadi $G(s)$ adalah strictly proper.

$(\Leftarrow)$ Misalkan $G(s)$ merupakan fungsi transfer yang strictly proper, sebutlah

$$
G(s)=\frac{L(s)}{d(s)}
$$

dimana

$$
\begin{aligned}
& L(s)=s^{n-1} L_{n-1}+\cdots+L_{0}, \\
& d(s)=s^{n}+d_{n-1} s^{n-1}+\cdots+d_{1} s+d_{0},
\end{aligned}
$$

untuk suatu $n \in \mathbb{N}$, dengan $L_{0}, L_{1}, \ldots, L_{n-1}$ adalah matriks-matriks berukuran $p \times$ m. Maka

$$
G(s)=\frac{s^{n-1} L_{n-1}+\cdots+L_{0}}{s^{n}+d_{n-1} s^{n-1}+\cdots+d_{0}}
$$




$$
=\frac{1}{d(s)}\left[\begin{array}{lllll}
L_{0} & L_{1} & \cdots & L_{n-2} & L_{n-1}
\end{array}\right]\left[\begin{array}{c}
I_{m} \\
s I_{m} \\
s^{2} I_{m} \\
\vdots \\
s^{n-1} I_{m}
\end{array}\right]
$$

dimana $I_{m}$ adalah matriks identitas berukuran $m \times m$. Misalkan

$$
Z=\frac{1}{d(s)}\left[\begin{array}{c}
I_{m} \\
s I_{m} \\
s^{2} I_{m} \\
\vdots \\
s^{n-1} I_{m}
\end{array}\right]=\left[\begin{array}{c}
Z_{1} \\
Z_{2} \\
\vdots \\
Z_{n-1} \\
Z_{n}
\end{array}\right]
$$

Dari (3.5) diperoleh

$$
Z_{1}=\frac{1}{d(s)} I_{m},
$$

yang mengakibatkan $Z_{i+1}=s Z_{i}$ dengan $i=1,2, \ldots, n-1$. Selain itu diperoleh juga

$$
Z_{n}=s^{n-1} Z_{1},
$$

yang dapat ditulis

$$
s Z_{n}=s^{n} Z_{1}
$$

Dari (3.6) diperoleh

$$
\begin{aligned}
I_{m} & =\left(d_{0}+d_{1} s+d_{2} s^{2}+\cdots+d_{n-1} s^{n-1}+s^{n}\right) Z_{1} \\
& =d_{0} Z_{1}+d_{1} s Z_{1}+d_{2} s^{2} Z_{1}+\cdots+d_{n-1} s^{n-1} Z_{1}+s^{n} Z_{1} \\
& =d_{0} Z_{1}+d_{1} Z_{2}+d_{2} Z_{3}+\cdots+d_{n-1} Z_{n}+s Z_{n}
\end{aligned}
$$

atau dapat ditulis

$$
s Z_{n}=I_{m}-d_{0} Z_{1}-d_{1} Z_{2}-d_{2} Z_{3}+\cdots-d_{n-1} Z_{n} .
$$

Selanjutnya (3.5) dapat ditulis menjadi

$$
\left[\begin{array}{c}
s Z_{1} \\
s Z_{2} \\
\vdots \\
s Z_{n-1} \\
s Z_{n}
\end{array}\right]=\left[\begin{array}{c}
Z_{2} \\
Z_{3} \\
\vdots \\
Z_{n} \\
-d_{0} Z_{1}-d_{1} Z_{2}-d_{2} Z_{3}+\cdots-d_{n-1} Z_{n}+I_{m}
\end{array}\right]
$$

atau

$$
s Z=A_{1} Z+B_{1},
$$

dimana

$$
A_{1}=\left[\begin{array}{cccccc}
0_{m} & I_{m} & 0_{m} & \cdots & 0_{m} & 0_{m} \\
0_{m} & 0_{m} & I_{m} & \cdots & 0_{m} & 0_{m} \\
\vdots & \vdots & \vdots & \ddots & \vdots & \vdots \\
0_{m} & 0_{m} & 0_{m} & \cdots & 0_{m} & I_{m} \\
-d_{0} I_{m} & -d_{1} I_{m} & -d_{2} I_{m} & \cdots & -d_{n-2} I_{m} & -d_{n-1} I_{m}
\end{array}\right]
$$




$$
B_{1}=\left[\begin{array}{c}
0_{m} \\
0_{m} \\
\vdots \\
0_{m} \\
I_{m}
\end{array}\right] .
$$

Dari (3.12) diperoleh $Z=\left(s I-A_{1}\right)^{-1} B_{1}$. Jadi, dengan memilih matriks $A_{1}$ seperti (3.13), $B_{1}$ seperti (3.14), dan

$$
C_{1}=\left[\begin{array}{llll}
L_{0} & L_{1} \cdots & L_{n-2} & L_{n-1}
\end{array}\right],
$$

maka $G(s)=C_{1}\left(s I-A_{1}\right)^{-1} B_{1}$, yang menunjukkan bahwa $A_{1}, B_{1}$, dan $C_{1}$ merupakan realisasi dari $G(s)$

Teorema 3.2. Realisasi dari $F(s)$ ada jika dan hanya jika $F(s)$ merupakan fungsi transfer yang entri-entrinya adalah polinomial.

Bukti $(\Rightarrow)$ Misalkan realisasi dari $F(s)$ ada, sebutlah realisasi tersebut adalah $N, B_{2}, C_{2}$ sedemikian sehingga $F(s)=C_{2}(s N-I)^{-1} B_{2}$. Akan ditunjukkan bahwa $F(s)$ merupakan fungsi transfer entri-entrinya adalah polinomial.

Ambil sebarang matriks

$$
\begin{aligned}
& N=\left[\begin{array}{ccccc}
n_{11} & n_{12} & n_{13} & \cdots & n_{1 n_{2}} \\
n_{21} & n_{22} & n_{23} & \cdots & n_{2 n_{2}} \\
n_{31} & n_{32} & n_{33} & \cdots & n_{3 n_{2}} \\
\vdots & \vdots & \vdots & \vdots & \vdots \\
n_{n_{2} 1} & n_{n_{2} 2} & n_{n_{2} 3} & \cdots & n_{n_{2} n_{2}}
\end{array}\right], B_{2}=\left[\begin{array}{ccccc}
b_{11} & b_{12} & b_{13} & \cdots & b_{1 m} \\
b_{21} & b_{22} & b_{23} & \cdots & b_{2 m} \\
\vdots & \vdots & \vdots & \vdots & \vdots \\
b_{n_{2} 1} & b_{n_{2} 2} & b_{n_{2} 3} & \cdots & b_{n_{2} m}
\end{array}\right] \\
& C_{2}=\left[\begin{array}{ccccc}
c_{11} & c_{12} & c_{13} & \cdots & c_{1 n_{2}} \\
c_{21} & c_{22} & c_{23} & \cdots & c_{2 n_{2}} \\
\vdots & \vdots & \vdots & \vdots & \vdots \\
c_{p 1} & c_{p 2} & c_{p 3} & \cdots & c_{p n_{2}}
\end{array}\right]
\end{aligned}
$$

dimana $N$ adalah matriks nilpoten.

Selanjutnya perhatikan bahwa

$$
\begin{aligned}
F(s)= & C_{2}(s N-I)^{-1} B_{2} \\
= & {\left[\begin{array}{cccc}
c_{11} & c_{12} & \cdots & c_{1 n_{2}} \\
\vdots & \vdots & \ddots & \vdots \\
c_{p 1} & c_{p 2} & \cdots & c_{p n_{2}}
\end{array}\right]\left[\begin{array}{cccc}
s n_{11}-1 & s n_{12} & \cdots & s n_{1 n_{2}} \\
s n_{21} & s n_{22}-1 & \cdots & s n_{2 n_{2}} \\
\vdots & \vdots & \ddots & \vdots \\
s n_{n_{2} 1} & s n_{n_{2} 2} & \cdots & s n_{n_{2} n_{2}}-1
\end{array}\right] } \\
& {\left[\begin{array}{cccc}
b_{11} & b_{12} & \cdots & b_{1 m} \\
\vdots & \vdots & \ddots & \vdots \\
b_{n_{2} 1} & b_{n_{2} 2} & \cdots & b_{n_{2} m}
\end{array}\right] }
\end{aligned}
$$

karena $N$ matriks nilpoten, maka entri-entri dari $F(s)$ dapat ditulis menjadi

$$
f_{i j}=f_{0}+f_{1} s+f_{2} s^{2}+\cdots+f_{n_{2}} s^{n_{2}},(i=1, \ldots, p ; j=1, \ldots, m) .
$$


Jadi $F(s)$ merupakan matriks yang berisikan polinomial-polinomial.

$(\Leftarrow)$ Misalkan $F(s)$ merupakan fungsi transfer dengan entri-entri polinomial. Tulis

$$
F(s)=\left[\begin{array}{ccccc}
f_{11} & f_{12} & f_{13} & \cdots & f_{1 m} \\
f_{21} & f_{22} & f_{23} & \cdots & f_{2 m} \\
\vdots & \vdots & \vdots & \ddots & \vdots \\
f_{p 1} & f_{p 2} & f_{p 3} & \cdots & f_{p m}
\end{array}\right]
$$

dengan

$$
f_{i j}=a_{i j}^{k_{j}} s^{k_{j}}+\cdots+a_{i j}^{1} s+a_{i j}^{0}, \quad(i=1, \ldots, p ; j=1, \ldots, m) .
$$

dimana $k_{j}$ adalah derajat tertinggi dari kolom ke- $j$ dari $F(s)$. Selanjutnya definisikan matriks

$$
\bar{S}=\operatorname{diag}\left[\bar{S}_{1}, \bar{S}_{2}, \cdots, \bar{S}_{m}\right], \bar{S}_{i}=\left[\begin{array}{c}
1 \\
s \\
\vdots \\
s^{k_{j}}
\end{array}\right] .
$$

Dengan menggunakan (3.16) dan (3.17), diperoleh

$$
F(s)=C_{2} \bar{S},
$$

dimana

$$
C_{2}=\left[\begin{array}{ccccccccc}
a_{11}^{0} & a_{11}^{1} & \cdots & a_{11}^{k_{1}} & \cdots & a_{1 m}^{0} & a_{1 m}^{1} & \cdots & a_{1 m}^{k_{m}} \\
a_{21}^{0} & a_{21}^{1} & \cdots & a_{21}^{k_{1}} & \cdots & a_{2 m}^{0} & a_{2 m}^{1} & \cdots & a_{2 m}^{k_{m}} \\
\vdots & \vdots & \cdots & \vdots & \ddots & \vdots & \vdots & \cdots & \vdots \\
a_{p 1}^{0} & a_{p 1}^{1} & \cdots & a_{p 1}^{k_{1}} & \cdots & a_{p m}^{0} & a_{p m}^{1} & \cdots & a_{p m}^{k_{m}}
\end{array}\right],
$$

dan $\bar{S}$ dapat ditulis sebagai

$$
\bar{S}=(s N-I)^{-1} B_{2},
$$

dimana

$$
\begin{aligned}
& N=\operatorname{diag}\left[N_{1}, N_{2}, \cdots, N_{m}\right], N_{j}=\left[\begin{array}{ccccc}
0 & 0 & \cdots & 0 & 0 \\
1 & 0 & \cdots & 0 & 0 \\
0 & 1 & \cdots & 0 & 0 \\
\vdots & \vdots & \ddots & 0 & 0 \\
0 & 0 & \cdots & 1 & 0
\end{array}\right], \\
& B_{2}=\operatorname{diag}\left[b_{1}, b_{2}, \ldots, b_{m}\right], b_{j}=\left[\begin{array}{c}
-1 \\
0 \\
\vdots \\
0
\end{array}\right],
\end{aligned}
$$

$N_{j} \in \mathbb{R}^{\left(k_{j}+1\right) \times\left(k_{j}+1\right)}$ dan $b_{j} \in \mathbb{R}^{\left(k_{j}+1\right) \times m}$, untuk $j=1, \ldots, m$. Jadi, dengan memilih matriks $N$ seperti (3.21), $B_{2}$ seperti (3.22), dan $C_{2}$ seperti (3.19), maka $F(s)=$ $C_{2}\left(s N-I_{n_{2}}\right)^{-1} B_{2}$, yang menunjukkan bahwa $N, B_{2}, C_{2}$ merupakan realisasi dari $F(s)$. 


\section{Contoh Penentuan Realisasi Fungsi Transfer}

Tentukan realisasi dari

$$
T(s)=\left[\begin{array}{cc}
\frac{s^{4}-s^{3}-5 s^{2}-2 s+2}{s^{2}-2 s-3} & s+1 \\
\frac{2 s^{3}-4 s^{2}-5 s}{s^{2}-2 s-3} & \frac{s^{3}-3 s^{2}+s-2}{s-3}
\end{array}\right] .
$$

Penyelesaian.

$T(s)$ dapat ditulis menjadi

$$
\begin{aligned}
T(s) & =G(s)+F(s) \\
& =\left[\begin{array}{ll}
\frac{s+2}{s^{2}-2 s-3} & 0 \\
\frac{s}{s^{2}-2 s-3} & \frac{1}{s-3}
\end{array}\right]+\left[\begin{array}{cc}
s^{2}+s & s+1 \\
2 s & s^{2}+1
\end{array}\right]
\end{aligned}
$$

Misalkan

$$
G(s)=\left[\begin{array}{cc}
\frac{s+2}{s^{2}-2 s-3} & 0 \\
\frac{s}{s^{2}-2 s-3} & \frac{1}{s-3}
\end{array}\right]
$$

dan

$$
F(s)=\left[\begin{array}{cc}
s^{2}+s & s+1 \\
2 s & s^{2}+1
\end{array}\right]
$$

Berdasarkan (4.2), diperoleh polinomial bersama dari penyebutnya sebagai berikut

$$
d(s)=s^{2}-2 s-3
$$

sehingga

$$
A_{1}=\left[\begin{array}{llll}
0 & 0 & 1 & 0 \\
0 & 0 & 0 & 1 \\
3 & 0 & 2 & 0 \\
0 & 3 & 0 & 2
\end{array}\right], B_{1}=\left[\begin{array}{ll}
0 & 0 \\
0 & 0 \\
1 & 0 \\
0 & 1
\end{array}\right]
$$

Dari (4.2) dan (4.4) diperoleh

$$
d(s) G(s)=\left[\begin{array}{cc}
s+2 & 0 \\
s & s+1
\end{array}\right] .
$$

Persamaan (4.5) dapat ditulis menjadi

$$
d(s) G(s)=\left[\begin{array}{ll}
2 & 0 \\
0 & 1
\end{array}\right]+\left[\begin{array}{ll}
1 & 0 \\
1 & 1
\end{array}\right] s,
$$

sehingga

$$
C_{1}=\left[\begin{array}{llll}
2 & 0 & 1 & 0 \\
0 & 1 & 1 & 1
\end{array}\right]
$$

Selain itu, berdasarkan (4.3) terlihat bahwa derajat tertinggi dari kolom pertama $F(s)$ adalah $k_{1}=2$ dan derajat tertinggi dari kolom kedua adalah $k_{2}=2$, sehingga 
diperoleh

$$
C_{2}=\left[\begin{array}{llllll}
0 & 1 & 1 & 1 & 1 & 0 \\
0 & 2 & 0 & 1 & 0 & 1
\end{array}\right], N=\left[\begin{array}{llllll}
0 & 0 & 0 & 0 & 0 & 0 \\
1 & 0 & 0 & 0 & 0 & 0 \\
0 & 1 & 0 & 0 & 0 & 0 \\
0 & 0 & 0 & 0 & 0 & 0 \\
0 & 0 & 0 & 1 & 0 & 0 \\
0 & 0 & 0 & 0 & 1 & 0
\end{array}\right], B_{2}=\left[\begin{array}{ccc}
-1 & 0 \\
0 & 0 \\
0 & 0 \\
0 & -1 \\
0 & 0 \\
0 & 0
\end{array}\right] .
$$

\section{Ucapan Terima Kasih}

Penulis mengucapkan terima kasih kepada Bapak Dr. Muhafzan, Bapak Dr. Admi Nazra, Bapak Dr. Syafrizal Sy, Bapak Dr. Mahdhivan Syafwan, dan Ibu Dr. Yanita yang telah memberikan masukan dan saran, sehingga tulisan ini dapat diselesaikan dengan baik.

\section{Daftar Pustaka}

[1] Duan, G. R. 2010. Analysis and Design of Descriptor Linear Systems. Springer. New York.

[2] Hendricks, E, Jannerup, O dan Sensen, P.H. 2008. Linear Systems Control. Springer. Verlag Berlin Heidelberg.

[3] Sinha, A. 2007. Linear Systems Optimal and Robust Control. CRC Press. New York. 